\title{
El autoexamen de mama: Traslación de la evidencia a la práctica clínica
}

\author{
Breast self-examination: Translating evidence into clinical practice
}

\section{Sr.Editor:}

El autoexamen de mama es una práctica que ha sido promovida desde la década de los cincuenta como un método de cribado para el diagnóstico temprano del cáncer de mama con el fin de disminuir la mortalidad (1).

En el articulo "Nivel de conocimiento y frecuencia de autoexamen de mama en alumnos de los primeros años de la carrera de Medicina" publicado en el fascículo 4 volumen 26 (octubre - diciembre 2015), Se explora el conocimiento de los alumnos de una facultad de medicina sobre el autoexamen de mama (2). Sin embargo existe evidencia acumulada de que esta es una práctica que no genera beneficios; no diminuye morbimortalidad y que puede aumentar procedimientos innecesarios en la población que es sometida a esta práctica.

Al respecto existe una revision del grupo Cochrane, donde se incluye 388535 mujeres; para determinar si el cribado mediante el autoexamen de mama reduce la morbilidad y mortalidad por cáncer de mama (3), en la que no se encontraron diferencias estadísticamente significativas entre el grupo de cribado y el grupo control. El Riesgo Relativo (RR) fue 1,05 (IC 95\% 0,90 a 1,24); de las 587 muertes por cáncer de mama, 292 fueron en el grupo de autoexamen y 295 en el grupo control. Sin embargo, se encontró casi el doble de biopsias (3406 biopsias) con resultados benignos realizadas en el grupo de cribado en comparación con el grupo de control (1856 biopsias), el RR fue de 1,88 (1,77 a 1,99) con IC del 95\%. Estos hallazgos podrian interpretarse como una tendencia la pruebas inncesarias en el grupo sometido a cribaje.

En este sentido la Sociedad Americana de Cáncer en su actualización 2015 se mantiene en no incluir como recomendación el autoexamen de mama para la detección del cáncer de mama (4). Así también las recomendaciones de la U.S. Preventive Services Task Force (USPSTF) incluyen no aconsejar la práctica del autoexamen de mama (5).

Es importante dada la evidencia actual, reflexionar sobre las consecuencias del sobrediagnóstico, sobretratamiento y sobretamizaje, que pueden incluir posibles alteraciones de las capacidades culturales e individuales para hacer frente a la enfermedad, el dolor y la muerte; aumento de la ansiedad, miedo y la percepción de la enfermedad cuando realmente no existe y un incremento de procedimientos o intervenciones innecesarias (6).

La tecnología ha mejorado el diagnóstico y tratamiento de numerosas enfermedades en los últimos 30 años. Las prácticas preventivas, incluyendo exámenes de despistaje de ciertos tipos de cáncer, han demostrado beneficios para la salud de la población; sin embargo, no todas las actividades preventivas son igual de efectivas y algunas pueden no tener sustento en la evidencia científica actual (6). Cuando hablamos de prevención es pertinente tener en cuenta

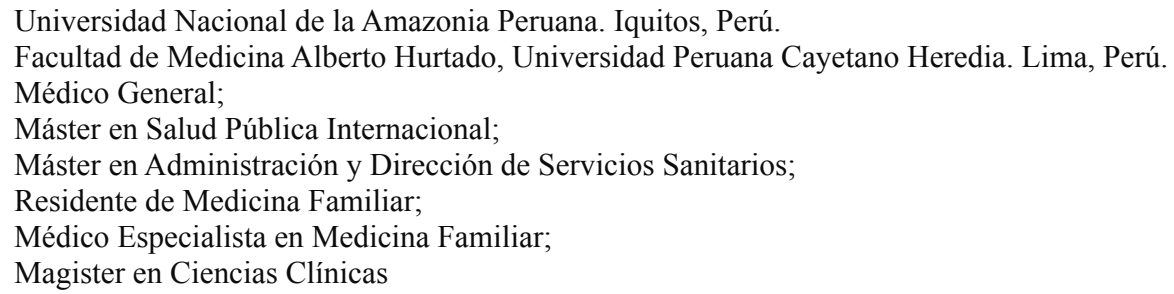


la prevención cuaternaria, definida como el conjunto de actividades que intentan evitar, reducir y paliar el perjuicio provocado por la intervención médica enmarcada en el principio de "primum non nocere" (7-9).

El interfaz entre la traslación de la evidencia científica a la práctica clínica o a la elaboración de políticas de salud en el Perú debería ser cada vez menor (10). En tanto las instituciones y profesionales de salud que aún promueven la práctica del autoexamen de mama están obligados a informar al paciente de los posibles daños que esta intervención produce.

\section{Gustavo Silva-Paredes ${ }^{\text {a,b,c, }}$ Pablo Ruíz Beteta ${ }^{1, d}$, María Sofía Cuba Fuentes ${ }^{2, e, f}$}

\section{REFERENCIAS BIBLIOGRÁFICAS}

1. Thornton H, Pillarisetti RR. Breast awareness and breast self-examination are not the same. What do these terms mean? Why are they confused? What can we do? Eur J Cancer. 2008; 44(15):2118-21.

2. Carrillo-Larco, RM et al. Nivel de conocimiento y frecuencia de autoexamen de mama en alumnos de los primeros años de la carrera de Medicina. Rev Med Hered, oct-dic 2015; 26(4), (citado 8 de julio 2016). Disponible en: http://www.scielo.org.pe/ scielo.php?script=sci_arttext\&pid=S1018-130X2015 000400002\&lng $=$ es\&nrm=iso

3. Kösters JP, Gøtzsche PC. Regular self-examination or clinical examination for early detection of breast cancer. Cochrane Database of Systematic Reviews. 2003; 2: CD003373. DOI: 10.1002/14651858. CD003373.

4. Oeffinger KC, Fontham EH, Etzioni R, et al. Breast cancer screening for women at average risk: 2015 guideline update from the american cancer society. JAMA. 2015; 314(15):1599-614.

5. Kühlein T, Sghedoni D, Visentin G, Guervas J, Jamoulle M. Quartäre Prävention-eine Aufgabe für Hausärzte. Primary Care. 2010; 10 (18): 350-4.
6. Blech J. Los inventores de enfermedades: como nos convierten en pacientes. Barcelona: Imago Mundi; 2005.

7. Gérvas Camacho J, Gavilán Moral E, Jiménez de García L. Prevención cuaternaria: es posible (y deseable) una asistencia sanitaria menos dañina. Actualización en Medicina de Familia. 2012; 8: 3127.

8. De Vito E. Prevención cuaternaria, un término aún no incluido entre los MESH. Medicina (Buenos Aires). 2013; 73(2):187-90.

9. Bentzen N (Editor). Wonca Dictionary of General/ Family Practice. Copenhagen: Maanedsskrift for Praktisk Laegegerning; 2003. p. 14.

10.Rychetnik L, Bauman A, Laws R, et al. Translating research for evidence-based public health: key concepts and future directions. J Epidemiol Community Health. 2012; 66(12):1187-92.

Recibido: 08/07/2016 